\title{
MRI versus Ultrasonography to Assess Meniscal Abnormalities in Acute Knees
}

James L. Cook, DVM, PhD ${ }^{1,2}$ Cristi R. Cook, DVM, MS ${ }^{1}$ James P. Stannard, MD ${ }^{2}$ Gavin Vaughn, MD Nichole Wilson, RN ${ }^{2}$ Brandon L. Roller, MD, PhD ${ }^{4}$ Aaron M. Stoker, MS, PhD ${ }^{1}$

Prakash Jayabalan, MD, $\mathrm{PhD}^{5}$ Moses Hdeib, MD, $\mathrm{PhD}^{6} \quad$ Keiichi Kuroki, DVM, $\mathrm{PhD}^{1}$

${ }^{1}$ Comparative Orthopaedic Laboratory, University of Missouri, Columbia, Missouri

2 Missouri Orthopaedic Institute, Department of Orthopaedic Surgery, University of Missouri, Columbia, Missouri

3 Department of Orthopaedic Surgery and Rehabilitation, Appalachian Regional Orthopaedic and Sports Medicine Center, Boone, North Carolina

${ }^{4}$ Arthrex Inc. Naples, Florida

${ }^{5}$ Department of Physical Medicine and Rehabilitation, University of

Pittsburgh Medical Center, Pittsburgh, Pennsylvania

${ }^{6}$ Department of Diagnostic Medical Ultrasound, University of

Missouri, Columbia, Missouri

J Knee Surg 2014;27:319-324.
Address for correspondence James L. Cook, DVM, PhD, Comparative Orthopaedic Laboratory, University of Missouri, Missouri Orthopaedic Institute, 1100 Virginia Avenue, Columbia, MO 65212 (e-mail: cookjl@health.missouri.edu).

\section{Abstract \\ Keywords \\ - ultrasonography \\ - magnetic resonance imaging \\ - meniscus \\ - knee \\ - arthroscopy}

While magnetic resonance imaging (MRI) is often considered the "gold standard" diagnostic imaging modality for detection of meniscal abnormalities, it is associated with misdiagnosis in as high as $47 \%$ of cases, is costly, and is not readily available to a large number of patients. Ultrasonographic examination of the knee has been reported to be an effective diagnostic tool for this purpose with the potential to overcome many of the shortcomings of MRI. The purpose of this study is to determine the clinical usefulness of ultrasonography for diagnosis of meniscal pathology in patients with acute knee pain and compare its diagnostic accuracy to MRI in a clinical setting. With Institutional Review Board approval, patients $(n=71)$ with acute knee pain were prospectively enrolled with informed consent. Preoperative MRI (1.5 T) was performed on each affected knee using the hospital's standard equipment and protocols and read by faculty radiologists trained in musculoskeletal MRI. Ultrasonographic assessments of each affected knee were performed by one of two faculty members trained in musculoskeletal ultrasonography using a 10 to $14 \mathrm{MHz}$ linear transducer. Arthroscopic evaluation of affected knees was performed by one of three faculty orthopedic surgeons to assess and record all joint pathology, which served as the reference standard for determining presence, type, and severity of meniscal pathology. All evaluators for each diagnostic modality were blinded to all other data. Data were collected and compared by a separate investigator to determine sensitivity (Sn), specificity (Sp), positive predictive value (PPV), negative predictive value (NPV), correct classification rate (CCR), likelihood ratios $(\mathrm{LR}[+]$ and $\mathrm{LR}[-])$, and odds ratios. Preoperative ultrasonographic assessment of meniscal pathology was associated with $\mathrm{Sn}=91.2 \%$, Sp received

December 11, 2013

accepted

December 18, 2013

published online

January 28, 2014
Copyright $\odot 2014$ by Thieme Medical Publishers, Inc., 333 Seventh Avenue, New York, NY 10001, USA. Tel: +1(212) 584-4662.
DOI http://dx.doi.org/ 10.1055/s-0034-1367731. ISSN 1538-8506. 
$=84.2 \%, \mathrm{PPV}=94.5 \%, \mathrm{NPV}=76.2 \%, \mathrm{CCR}=89.5 \%, \mathrm{LR}(+)=5.78$, and $\mathrm{LR}(-)=0.10$. Preoperative MRI assessment of meniscal pathology was associated with $\mathrm{Sn}=91.7 \%$, $\mathrm{Sp}=66.7 \%, \quad \mathrm{PPV}=84.6 \%, \quad \mathrm{NPV}=80.0 \%, \quad \mathrm{CCR}=81.1 \%, \quad \operatorname{LR}(+)=2.75, \quad$ and $\quad \mathrm{LR}$ $(-)=0.13$. Ultrasonography was two times more likely than MRI to correctly determine presence or absence of meniscal pathology seen arthroscopically in this study. Ultrasonography is a useful tool for diagnosis of meniscal pathology with potential advantages over MRI. Based on these data and available portable equipment, ultrasonography could be considered for use as a point-of-injury diagnostic modality for meniscal injuries.

Nearly 1 million meniscal injuries occur in the United States annually. ${ }^{1}$ Currently, meniscal pathology is most often diagnosed based on history, clinical examination, magnetic resonance imaging (MRI), and/or arthroscopic visualization. ${ }^{2-22}$ Early and accurate diagnosis of meniscal pathology is vital for determining type and timing of treatment, as well as prognosis for return to function in the short term and degree of morbidity in the long term. In the United States, preoperative diagnosis of meniscal pathology is largely based on MRI of the affected knee. Diagnostic MRI may not be performed until weeks or even months after injury, and while MRI is often considered the "gold standard" diagnostic imaging modality for detection of meniscal abnormalities, it is associated with misdiagnosis in 14 to $47 \%$ of cases. ${ }^{7,10,13,14,21,22}$ MRI is also costly and is not readily available to a large number of patients for either financial or logistical reasons, or both. Because early and accurate diagnosis of meniscal pathology is imperative for treatment planning and prognostication, this imaging modality may not be efficient and effective for optimal management of patients with meniscal pathology.

Ultrasonographic examination of the knee shows promise for being an effective diagnostic tool for assessing meniscal pathology with the potential to overcome many of the shortcomings of MRI. Ultrasonography has been used for diagnosis of meniscal pathology in veterinary medicine for more than a decade with reported sensitivities and specificities as high as 90 and $92.9 \%$, respectively. ${ }^{23}$ Studies in human patients report sensitivities ranging from 30 to $100 \%$ and specificities ranging from 71.4 to $98 \%$ for sonographic diagnosis of meniscal pathology. ${ }^{2-6,8,9,12,15-20}$ The most recent studies report the highest sensitivities, specificities, and correct classification rates (CCRs), likely as a result of improvements in technology, training, and experience. Based on the recent results, the costs and availability, the portability of the equipment, and the safety associated with ultrasonography, the authors of these studies concluded that ultrasonography is a clinically useful diagnostic technique for meniscal pathology. However, the capabilities of ultrasonography for clinical diagnosis of meniscal abnormalities in patients have not been fully evaluated. Therefore, the objective of this study was to determine the usefulness of ultrasonography for diagnosis of meniscal pathology in patients with acute knee pain and compare its diagnostic accuracy to MRI in a clinical setting.

\section{Methods}

With Institutional Review Board approval, patients $(n=71)$ with acute knee pain were prospectively enrolled through informed consent. Patients were included in the study if they were 14 years of age and older, they presented with acute knee pain, and they had a high probability for requiring surgical intervention for diagnosis and/or treatment of the affected knee(s). Patients were excluded when these criteria were not met or informed consent was not granted and documented.

Epidemiologic data, sonographic imaging findings, MRI reports, and surgery reports and images (when available) were recorded in the patients' medical records and the dedicated database for the study. Data were only retrieved for analysis.

Preoperative MRI was performed on each affected knee using the hospital's standard equipment and protocols and read by faculty radiologists trained in musculoskeletal MRI. All MRIs were performed using 1.5 T units with dedicated knee coils. Sequences included were at the discretion of the attending radiologists based on their preferences and standard-ofcare clinical practice. The attending radiologist reviewed the MRI and subjectively classified each meniscus as normal, torn (with description of location and type when possible), or showing abnormal signal, but not torn. MRI reports were entered into the medical record and data specific to meniscal findings were entered into a separate database by another investigator blinded to all other diagnostic findings.

At a separate appointment, each patient underwent ultrasonographic examination of the affected knee conducted by one ultrasonographer blinded to patient history, physical examination findings, and MRI findings. Ultrasonographic examination was performed by one of two investigators trained in musculoskeletal ultrasonography using a portable ultrasound machine (Logiq i, GE Health Care, Milwaukee, WI) with a 10 to $14 \mathrm{MHz}$ linear transducer. Each patient was initially placed supine with the knee extended, so that the anterior horns of the menisci could be examined. The knee was then flexed to 90 degrees and the probe rotated laterally to examine the anterior cruciate ligament $(A C L)$ (this is a dynamic examination with the knee being serially extended). The patient was then placed prone so the posterior horns of each meniscus could be 


$\begin{array}{llll} & \text { Mizzou Meniscal Ultrasound Assessment } & \text { Form } \\ \text { Patient ID__nate: } & \text { Date } & & \\ \text { Ultrasonographer } & \text { Knee: } & \mathbf{R} & \mathbf{L}\end{array}$

Medial

\begin{tabular}{|l|l|l|l|}
\hline & Anterior & Body & Posterior \\
\hline Displacement & & & \\
\hline Echogenecity & & & \\
\hline Shape & & & \\
\hline Fluid & & & \\
\hline
\end{tabular}

Score each area and each category as follows:

$0=$ normal

$1=$ mild pathology

$2=$ moderate pathology

3 = severe pathology

Ultrasonographic Diagnosis:

Lateral

\begin{tabular}{|l|l|l|l|}
\hline & Anterior & Body & Posterior \\
\hline Displacement & & & \\
\hline Echogenecity & & & \\
\hline Shape & & & \\
\hline Fluid & & & \\
\hline
\end{tabular}

Score each area and each category as follows:

$0=$ normal

$1=$ mild pathology

2 = moderate pathology

3 = severe pathology

Ultrasonographic Diagnosis:

Miscellaneous Findings / Comments:

Displacement - abaxial meniscal margin extends abaxial to the cortical margins of femur and tibia

Echogenicity - meniscal parenchyma not uniformly hyperechoic with uniform echotexture

Shape - cross section of meniscus is not a sharp, well defined triangle that parallels the apposing articular surfaces of femoral and tibial condyles

Fluid - any fluid associated with the meniscus that is more than a uniform anechoic fluid line 1$2 \mathrm{~mm}$ interposed between superior and inferior margins of meniscus and femoral and tibial surfaces, respectively, or any marked asymmetry in the fluid lines or fluid pockets abaxial to the peripheral margin of the meniscus

Fig. 1 Ultrasound assessment form developed for evaluations.

examined. Each meniscus was evaluated for displacement, echogenicity, shape, and associated effusion (-Fig. 1). Scores were used for determining strength of correlations between ultrasonographic findings and basic science data for another portion of this research. The presence or absence of meniscal pathology was determined based on the ultrasonographer's overall assessment using the four criteria in the standardized assessment protocol to subjectively determine deviations from normal. Location of the tear was determined, however, characterization of type of tear was not attempted. Rather, the goal was to determine whether or not meniscal pathology warranting surgical intervention was present. Findings consistent with joint effusion, synovial thickening, and osteophytosis were also recorded. Ultrasonography reports were entered into the medical record and data specific to meniscal findings were entered into a separate database by another investigator blinded to all other diagnostic findings.

Based on indication and with informed patient consent, complete arthroscopic examination of affected knees was performed by one of three faculty orthopedic surgeons to assess and record all joint pathology, which served as the reference standard for determining presence, type, and severity of meniscal pathology present. Both menisci were assessed by visualization and palpation with an arthroscopic probe and all findings were subjectively described and recorded. Arthroscopy reports were entered into the medical record and data specific to meniscal findings were entered into a separate database by another investigator blinded to all other diagnostic findings.

Arthroscopic data were used as the reference standard for statistical analyses. Data were compiled and analyzed by a 
separate investigator to determine sensitivity, specificity, positive predictive value (PPV), negative predictive value (NPV), correct classification rate (CCR), likelihood ratios (LR $[+], \operatorname{LR}[-])$, and odds ratios.

\section{Results}

Patients included in the study ranged in age from 15 to 73 years with a mean age of 37.2 years. Forty patients (56\%) were male and 31 (44\%) were female.

Based on arthroscopic evaluation, 59 patients had identifiable meniscal pathology (49 medial, 18 lateral, 4 both) and 12 patients had no definitive meniscal pathology identified.

- Table 1 provides sensitivity, specificity, PPV, NPV, CCR, and likelihood ratios for MRI and ultrasonographic assessments of meniscal pathology. Based on odds ratio analysis, ultrasonography was two times more likely than MRI to correctly determine presence or absence of meniscal pathology diagnosed based on arthroscopic assessments (-Fig. 2).

For MRI, incorrect diagnoses involved missing lateral meniscal degeneration, missing medial radial tears, missing medial longitudinal tears, missing lateral tears when both menisci were torn, incorrectly reporting lateral tears as medial tears, and reporting tears not identified arthroscopically.

For ultrasonography, incorrect diagnoses involved missing medial and lateral radial tears, missing lateral tears when both menisci were torn, and reporting tears not identified arthroscopically.

\section{Discussion}

This study produced data that are in agreement with previous work suggesting that ultrasonography is a useful tool for diagnosis of meniscal pathology with potential advantages over MRI. By using a standardized assessment system for meniscal ultrasonography, consistent and accurate information was obtained. This assessment system provides ultrasonographers with a methodology for progressing efficiently along the learning curve of this technique to obtain results similar to ours in clinical practice. In this prospective study, ultrasonographic assessment was two times more likely to correctly determine the presence or absence of meniscal pathology in patients with acute knee pain with a CCR of $89.5 \%$ compared with $81.1 \%$ for MRI. This means that MRI is "wrong" almost twice as often as ultrasound for determining the presence or absence of meniscal pathology as assessed in this study.

Importantly, the greatest statistical differences between the two techniques were noted for specificity and positive likelihood ratios. These differences provide further evidence for the benefits of ultrasonography for being associated with a higher probability to be correct in determining the absence of meniscal pathology and differentiating patients with meniscal pathology from those without meniscal pathology. Based on the extremely large volume of meniscal injuries treated each year-approximately 1 million per year in the United States alone ${ }^{1}$-and the fact that the majority of these injuries require surgical intervention, the authors suggest these findings are of critical clinical importance.

The advantages of ultrasound as a diagnostic test would translate into the avoidance of unnecessary surgeries and related morbidity, as well as profound savings in related health care costs. Similarly, financial benefits related to costs for diagnostic imaging could also be realized. At the time of study initiation, our institution's charges were $\$ 384$ to perform and read an ultrasonographic study of the knee and $\$ 1,211$ for MRI of the knee as described. Coupled with the high safety of ultrasound and its relative comfort for patients with claustrophobia, phonophobia, or magnet-sensitive implants, these features make ultrasound very attractive for clinical use in diagnostic imaging for meniscal pathology.

Another potential advantage of ultrasonography for diagnosis of meniscal pathology is related to the portability of current equipment. High-quality ultrasound machines are available in laptop and hand-held versions. A portable, laptop version was used for imaging in the present study. This factor provides the potential for point-of-injury diagnostic imaging, which for athletic activities includes the potential for "on-thefield" diagnostics and decision making. As an example, American football has one of the highest rates of injury associated with an athletic activity with more than one-third of all football injuries involving the knee. ${ }^{24-26}$ Football players are nearly six times more likely to suffer knee injuries requiring surgery than the general population. ${ }^{27} \mathrm{~A}$ study that evaluated athletes at the National Football League Combine for incoming rookies described a meniscal tear/injury incidence of 12.4 per 100 players, and indicated meniscectomy as the most common procedure performed on these athletes with an incidence of 10.3 meniscectomies per 100 players. $^{28}$ Based on current protocols, there is typically at least a 24- to 72-hour delay before diagnostic imaging in the form of MRI is performed, which renders the patient and medical staff unable to make an informed decision regarding treatment and prognosis, and potentially allows for further damage to occur. This same scenario could be multiplied by the thousands by application to male and female soccer players around the world-as well as any other athletic activity for people of all ages-many of whom have no access

Table 1 Sensitivity, specificity, PPV, NPV, CCR, LR+, and LR - for MRI and ultrasonographic assessments of meniscal pathology

\begin{tabular}{|l|l|l|l|l|l|l|l|}
\hline Modality & Sensitivity & Specificity & PPV & NPV & CCR & LR+ & LR - \\
\hline Ultrasound & $91.2 \%$ & $84.2 \%$ & $94.5 \%$ & $76.2 \%$ & $89.5 \%$ & 5.78 & 0.10 \\
\hline MRI & $91.7 \%$ & $66.7 \%$ & $84.6 \%$ & $80.0 \%$ & $81.1 \%$ & 2.75 & 0.13 \\
\hline
\end{tabular}

Abbreviations: CCR, correct classification rate; LR+, positive likelihood ratio; LR-, negative likelihood ratio; NPV, negative predictive value; PPV, positive predictive value. 

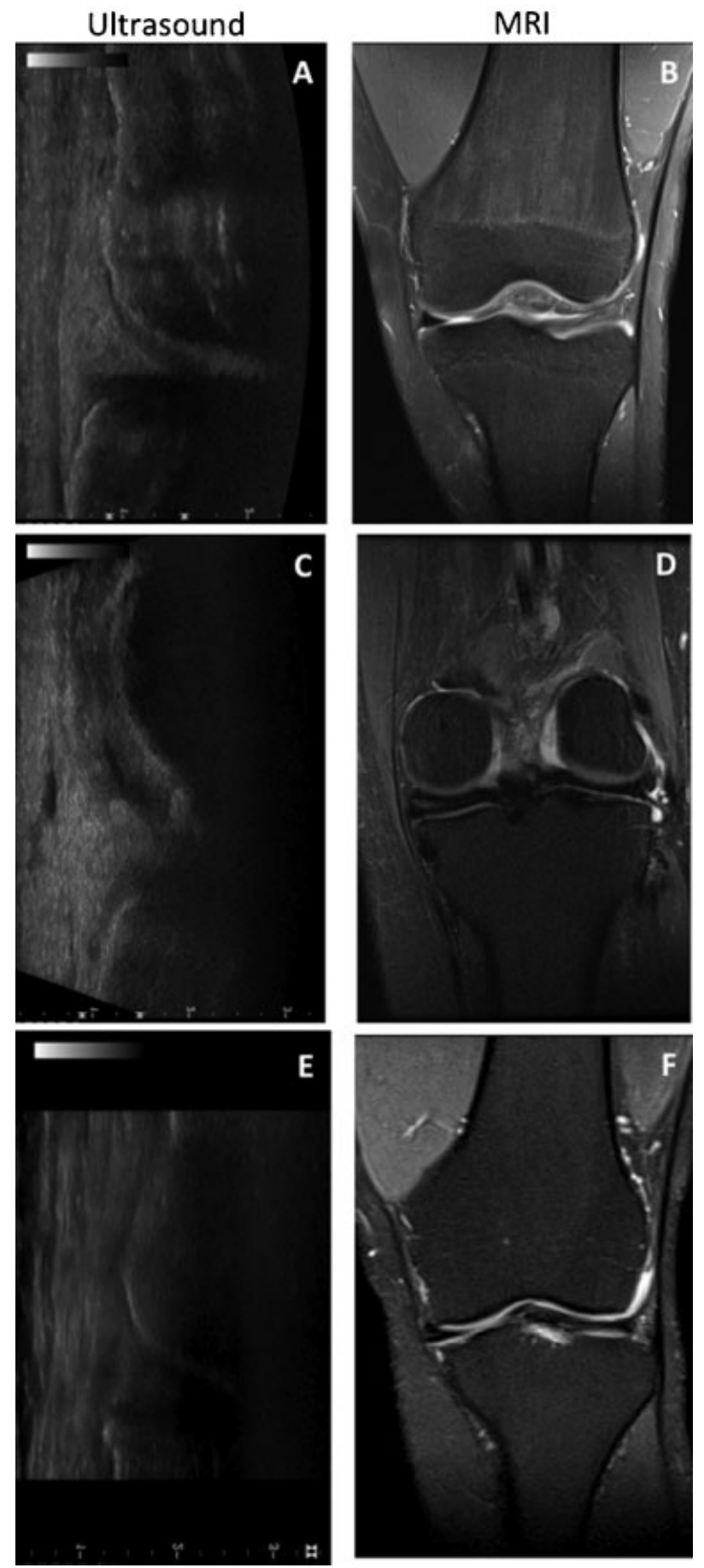

Fig. 2 Representative images from cases included in this study showing normal $(\mathrm{A}, \mathrm{B})$ and pathologic $(\mathrm{C}-\mathrm{F})$ menisci based on arthroscopic assessment, which were all correctly diagnosed by ultrasonography while one $(F)$ was incorrectly diagnosed by magnetic resonance imaging.

(logistical and/or financial) to MRI. Ultrasonography provides a portable, cost effective, and accurate tool for earlier diagnosis for these patients so optimal management can be efficiently pursued.

It should be pointed out that these findings apply only to diagnosis of meniscal pathology. While ultrasonographic assessment of the knee can include evaluation of medial collateral ligament, lateral collateral ligament, posterior lateral corner, joint capsule and limited regions of the ACL, PCL, and articular cartilage, assessment of these structures was not included in the present study and there are aspects of pathology of the knee that cannot be determined using ultrasonography. Therefore, it should be emphasized that MRI has advantages over ultrasonography for comprehensive diagnostic imaging of the knee and ultrasonographic assessment cannot be used alone to completely assess the injured knee. Knee ultrasonography as described in this study can only be applied to targeted evaluation of the menisci.

The limitations of the present study primarily involve the number and type of patients included and the relative inequity among imagers performing the two different diagnostic imaging techniques. Certainly, a larger number and spectrum of patients need to be included in a multicenter trial that validates the findings of this study before conclusive arguments regarding preference for use of ultrasonography over MRI for diagnosis of meniscal pathology can be made. Similarly, additional imagers-perhaps using a standardized and optimized MRI protocol-would need to be included for both modalities to ensure that these data are broadly applicable. It could also be argued that higher strength $(3 \mathrm{~T})$ magnets with advanced software would likely provide improved diagnostic capabilities and compare more favorably with ultrasonography for assessment of meniscal pathology. However, that level of MRI technology is not widely available for routine clinical use at this time and therefore would not provide a "real life" standard-of-care comparison for broad application.

At this point in development, the ultrasonographic assessment methodology we employ is not based on quantitative or even semiquantitative measures. While scores were assigned in each of the four categories included in the standardized system, those scores were used for determining strength of correlations in other aspects of this research and not used as thresholds for presence or severity of meniscal pathology. However, the use of the standardized criteria and technique described resulted in consistent and accurate results from two different ultrasonographers in terms of correctly classifying presence or absence and location of meniscal pathology in patients presenting for acute knee pain.

In summary, this study provides evidence for the use of standardized ultrasonographic assessment of the knee as a useful tool for diagnosis of meniscal pathology with potential advantages over MRI. Based on these data and available portable equipment, ultrasonography could be considered for use as a point-of-injury diagnostic modality for meniscal injuries.

\section{References}

1 Medical Data International; Newport Beach, CA; September 2003 2 Alizadeh A, Babaei Jandaghi A, Keshavarz Zirak A, Karimi A, Mardani-Kivi M, Rajabzadeh A. Knee sonography as a diagnostic test for medial meniscal tears in young patients. Eur J Orthop Surg Traumatol 2013;23(8):927-931 
3 Azzoni R, Cabitza P. Is there a role for sonography in the diagnosis of tears of the knee menisci? J Clin Ultrasound 2002;30(8):472-476

4 Casser HR, Sohn C, Kiekenbeck A. Current evaluation of sonography of the meniscus. Results of a comparative study of sonographic and arthroscopic findings. Arch Orthop Trauma Surg 1990;109(3): 150-154

5 Court-Payen M. Sonography of the knee: intra-articular pathology. J Clin Ultrasound 2004;32(9):481-490

6 De Maeseneer M, Lenchik L, Starok M, Pedowitz R, Trudell D, Resnick D. Normal and abnormal medial meniscocapsular structures: MR imaging and sonography in cadavers. AJR Am J Roentgenol 1998;171(4):969-976

7 Esmaili Jah AA, Keyhani S, Zarei R, Moghaddam AK. Accuracy of MRI in comparison with clinical and arthroscopic findings in ligamentous and meniscal injuries of the knee. Acta Orthop Belg 2005;71(2):189-196

8 Friedl W, Glaser F. Dynamic sonography in the diagnosis of ligament and meniscal injuries of the knee. Arch Orthop Trauma Surg 1991;110(3):132-138

9 Grifka J, Richter J, Gumtau M. [Clinical and sonographic meniscus diagnosis]. Orthopade 1994;23(2):102-111

10 Harper KW, Helms CA, Lambert HS III, Higgins LD. Radial meniscal tears: significance, incidence, and MR appearance. AJR Am J Roentgenol 2005;185(6):1429-1434

11 Karachalios T, Hantes M, Zibis AH, Zachos V, Karantanas AH, Malizos KN. Diagnostic accuracy of a new clinical test (the Thessaly test) for early detection of meniscal tears. J Bone Joint Surg Am 2005;87(5):955-962

12 Khan Z, Faruqui Z, Ogyunbiyi O, Rosset G, Iqbal J. Ultrasound assessment of internal derangement of the knee. Acta Orthop Belg 2006;72(1):72-76

13 Kornaat PR, Bloem JL, Ceulemans RY, et al. Osteoarthritis of the knee: association between clinical features and MR imaging findings. Radiology 2006;239(3):811-817

14 Laoruengthana A, Jarusriwanna A. Sensitivity and specificity of magnetic resonance imaging for knee injury and clinical application for the Naresuan University Hospital. J Med Assoc Thai 2012; 95(Suppl 10):S151-S157

15 Lee D, Bouffard JA. Ultrasound of the knee. Eur J Ultrasound 2001; 14(1):57-71
16 Mattli J, Holzach P, Soklic P. [Meniscus ultrasound-a reliable way for diagnosis of meniscus lesions?] Z Unfallchir Versicherungsmed 1993;1(Suppl 1):133-140

17 Najafi J, Bagheri S, Lahiji FA. The value of sonography with micro convex probes in diagnosing meniscal tears compared with arthroscopy. J Ultrasound Med 2006;25(5):593-597

18 Petersen LJ, Rasmussen OS. [Ultrasonography as a diagnostic method in suspected meniscal lesion of the knee. A prospective single blind study of 52 patients]. Ugeskr Laeger 1999;161(41): 5679-5682

19 Richter J, Grifka J, Fisseler-Eckhoff A, Müller KM, Krämer J. [Ultrasound morphologic criteria in evaluating meniscus changes-an experimental study]. Z Orthop Ihre Grenzgeb 1996;134(2): 137-143

20 Riedl S, Tauscher A, Kühner C, Göhring U, Sohn C, Meeder PJ. [3dimensional ultrasound in clinical diagnosis of meniscus lesions]. Ultraschall Med 1998;19(1):28-33

21 Vaz CE, Camargo OP, Santana PJ, Valezi AC. Accuracy of magnetic resonance in identifying traumatic intraarticular knee lesions. Clinics (Sao Paulo) 2005;60(6):445-450

22 Ververidis AN, Verettas DA, Kazakos KJ, Tilkeridis CE, Chatzipapas $\mathrm{CN}$. Meniscal bucket handle tears: a retrospective study of arthroscopy and the relation to MRI. Knee Surg Sports Traumatol Arthrosc 2006;14(4):343-349

23 Mahn MM, Cook JL, Cook CR, Balke MT. Arthroscopic verification of ultrasonographic diagnosis of meniscal pathology in dogs. Vet Surg 2005;34(4):318-323

24 Garrick JG, Requa RK. Injuries in high school sports. Pediatrics 1978;61(3):465-469

25 Maffulli N, Chan KM, Miao M, Fu FH, Kurosaka M. Athletic knee injuries. Similarities and differences between Asian and Western experience. Clin Orthop Relat Res 1996;323(323): 98-105

26 Saal JA. Common American football injuries. Sports Med 1991; 12(2):132-147

27 DeLee JC, Farney WC. Incidence of injury in Texas high school football. Am J Sports Med 1992;20(5):575-580

28 Brophy RH, Barnes R, Rodeo SA, Warren RF. Prevalence of musculoskeletal disorders at the NFL Combine-trends from 1987 to 2000. Med Sci Sports Exerc 2007;39(1):22-27 